\title{
Both stress experience and age determine the impairment or enhancement effect of stress on spatial memory retrieval
}

\author{
Y Yang, J Cao, W Xiong, J Zhang, Q Zhou, H Wei ${ }^{1}$, C Liang ${ }^{1}$, \\ J Deng ${ }^{2}$, T Li ${ }^{2}$, S Yang, and $\mathrm{L} \mathrm{Xu}$ \\ Laboratory of Learning and Memory, Kunming Institute of Zoology, The Chinese Academy of Science, Kunming, 650223, Republic of China \\ ${ }^{1}$ Kunming General Hospital, ChengDu area, Kunming, Republic of China \\ ${ }^{2}$ Yunnan Institute of Agriculture, Kunming, Republic of China \\ (Requests for offprints should be addressed to L Xu; Email: Ixu@vip.163.com)
}

\begin{abstract}
It has been documented that stress or glucocorticoids have conflicting effects on memory under different conditions. However, it is not fully understood why stress can either impair or enhance memory. Here, we have examined the performance of six age groups of Wistar rats in a water maze spatial task to evaluate the effects of stress under different conditions. We found that the impairment or enhancement effect of an 'elevated platform' (EP) stress on memory was dependent on previous stress experience and on age. EP stress impaired memory retrieval in water maze naive animals, but enhanced rather than impaired memory retrieval in young water maze stress-experienced animals. Furthermore, exogenously applied corticosterone or foot shock stress before water maze training prevented the
\end{abstract}

impairment of memory retrieval that should be induced by treatment with corticosterone or foot shock before the 'probe trial'. Again, memory retrieval was enhanced in young animals under these conditions, and this enhancement can be prevented by the glucocorticoid receptor antagonist RU 38486. Thus, glucocorticoid receptor activation not only induced impairment of memory but also increased the capacity of young animals to overcome a later stress. The present findings suggest that the effect of stress on memory can be switched from impairment to enhancement dependent on both stress experience and age.

Journal of Endocrinology (2003) 178, 45-54

\section{Introduction}

Extensive evidence shows that learning and memory is exquisitely sensitive to behavioral stress (McEwen \& Sapolsky 1995, McGaugh et al. 1996, de Quervain et al. 1998, Ferry et al. 1999). However, studies in animals and people have reported divergent findings, with stress producing an enhancement, impairment, or no effect on learning and memory (Shors et al. 1992, Diamond et al. 1999, de Kloet et al. 1999, Sapolsky 2000, Garcia 2001, Lupien \& Lepage 2001, Payne et al. 2002). A fundamental question arises as to when and how stress switches its effect on memory from impairment to enhancement. Several studies have suggested that the duration of stress and the receptor type are critical factors in the control of learning and memory (Flood et al. 1972, McEwen et al. 1995, Luine et al. 1996, Sandi et al. 1997, Shors \& Servatius 1997, Oitzl et al. 1998, Conrad et al. 1999, de Kloet et al. 1999). Other evidence has suggested that memory can be either impaired or enhanced by stress dependent on when stress is given, in context or out of context (Shors \& Servatius 1997, Garcia et al. 1998).
Some sorts of stress (such as adaptable and inadaptable stress) are unavoidable in daily life and the stress experience can be memorized for life. As hypothalamicpituitary-adrenal activity is different in normal ontogeny and the hippocampus plays an important role in feedback inhibition of the stress response (Bodnoff et al. 1995, McEwen \& Sapolsky 1995, Garcia 2001, Lupien \& Lepage 2001), the effect of stress on memory may be dependent on age and previous stress experience (Sandi et al. 1997, McEwen 2000). It is possible that if animals encounter acute stress abruptly, their memory will be severely impaired no matter what their age, whereas the stress effects on learning and memory could be very different if different aged groups of animals had previous stress experience.

Thus, we have studied the effect of behavioral stress on memory in a Morris water maze by three experimental protocols in which different aged groups of animals had previous experience of the water maze, and of 'elevated platform' (EP) and foot shock stress. We also examined the effect of corticosterone in order to mimic the effect of previous stress experience on memory. 


\section{Materials and Methods}

Animals

Experiments were carried out on male Wistar rats (inbred strain, Animal House Center, Kunming General Hospital, Kunming), aged from 4 to 76 weeks. Animals were group-housed with free access to water and food in an established animal house having a $12 \mathrm{~h}$ light: $12 \mathrm{~h}$ darkness cycle and a thermoregulated environment. The animal care and experimental protocol were approved by the Yunnan Health Department, China.

\section{Water maze}

The apparatus consisted of a circular pool $(250 \mathrm{~cm}$ diameter, $60 \mathrm{~cm}$ deep at the side) filled with water at $25 \pm 1{ }^{\circ} \mathrm{C}$ to a depth of $20 \mathrm{~cm}$, and the surface was covered with floating black resin beads. Yellow curtains were drawn around the pool $(50 \mathrm{~cm}$ from the pool periphery) which contained distinctive visual marks that served as cues.

\section{Training procedures}

Rats were trained in a conventional Morris water maze task (Morris 1984). The water maze was divided into four imaginary quadrants. Animals always faced the wall when they were placed into the maze from different starting positions (e.g. E, W, N, S, NE, NW, SE, SW).

Water maze stress experience Animals were allowed $180 \mathrm{~s}$ swimming in the water maze without the escape platform four times per day for five consecutive days. Water maze naive rats were handled four times per day for five consecutive days.

Thirty-two-trials training A submerged Perspex platform $(14 \mathrm{~cm} \times 14 \mathrm{~cm})$ was placed in the middle of one quadrant for all training trials. Each animal was trained for eight trials per day with inter-trial intervals of 20-40 min for four consecutive days. Four trials were defined as one training session. Animals were allowed to swim until they found the hidden platform and stayed there for $30 \mathrm{~s}$ before being picked up. The animals that failed to find the hidden platform in $180 \mathrm{~s}$ were guided to it.

Six-trials training The training procedure was similar to the thirty-two-trials training except only six trials were given with $30 \mathrm{~min}$ inter-trial intervals at six different starting positions that were equally distributed around the perimeter of the maze.

Probe trial This trial was initiated $30 \mathrm{~min}$ after the end of training and consisted of $180 \mathrm{~s}$ swimming without the escape platform.
Swimming paths for all training trials and the probe trial were monitored using an automatic tracking system. The average swimming speed was calculated from the total path lengths of the probe trial. Experiments were conducted between $0900 \mathrm{~h}$ and $1600 \mathrm{~h}$.

\section{Stress protocol}

Behavioral stress was evoked by placing the rats on an elevated platform (EP) in the middle of a brightly lit room for $30 \mathrm{~min}$ (Balfour \& Reid 1979, Xu et al. 1997) or by giving foot shocks $(\mathrm{F}, 0.8 \mathrm{~mA}$ for $1 \mathrm{~s}$ with a 5 -s inter-shock interval) in an operant test chamber (Med Associates Inc., St Albans, Herts, UK) (de Quervain et al. 1998).

\section{Experimental groups}

Thirty-two-trials training EP-W animals received EP stress before each training session (four trials were defined as one training session). W-EP animals received EP stress after each session. The probe trial was given $30 \mathrm{~min}$ after the final training trial or immediately after the final $30 \mathrm{~min}$ EP stress. Control animals received the same training as other groups without EP stress.

Six-trials training $6 t-E P$ animals received 30 min EP stress after the final trial, and the probe trial was given immediately after the EP stress. 6t/6EP animals received 30 min EP stress after each trial, and the probe trial was given immediately after the final EP stress. 6t-Cort animals received corticosterone $(5 \mathrm{mg} / \mathrm{kg}$, s.c.) immediately after the final trial, $30 \mathrm{~min}$ before the probe trial. Control animals received the same training as the other groups but without EP stress, F stress or corticosterone treatment. Cort-6t-Cort animals received corticosterone $(5 \mathrm{mg} / \mathrm{kg}$, s.c.) immediately before the first trial and again after the final trial. 6t-F animals received $\mathrm{F}$ stress immediately after the final trial, $30 \mathrm{~min}$ before the probe trial. F-6t-F animals received $\mathrm{F}$ stress immediately before the first trial and then after the final trial, $30 \mathrm{~min}$ before the probe trial. F-6t-EP animals received $\mathrm{F}$ stress immediately before the first trial and 30 min EP stress immediately after the final trial. The probe trial was given immediately after EP stress. Ru-6t animals received RU 38486 $(20 \mathrm{mg} / \mathrm{kg}$, s.c.) immediately before the first trial and the probe trial was given $30 \mathrm{~min}$ after the final trial. Ru-6t/6EP animals received RU 38486 (20 mg/kg, s.c.) immediately before the first trial and EP stress was given after each trial. The probe trial was given immediately after the final EP stress. Vehicle-treated animals received $0.2 \mathrm{ml}$ polyethylene glycol immediately before the first trial.

Corticosterone $(5 \mathrm{mg} / \mathrm{kg})$ and RU $38486(20 \mathrm{mg} / \mathrm{kg})$ were dissolved in polyethylene glycol and injected subcutaneously at a volume of $0 \cdot 2 \mathrm{ml}$. 


\section{Plasma levels of corticosterone}

Trunk blood was collected immediately after the probe trial. After centrifugation at $10000 \mathrm{~g}$ for $10 \mathrm{~min}$, the supernatant was stored at $-70{ }^{\circ} \mathrm{C}$ until assay. Levels of plasma corticosterone were determined with a rat corticosterone assay kit $\left.{ }^{125} \mathrm{I}\right]$ (Amersham Life Science). Statistical comparison was made using a $t$-test or repeated measures or one-way ANOVA followed by a least significance difference test (SPSS 10·0).

\section{Results}

EP stress enhanced spatial memory in young water maze stress-experienced rats

In the probe trial $30 \mathrm{~min}$ after the final trial or immediately after the final EP stress, water maze naive control rats spent significantly more time in the target quadrant than the 10to 76 -week-old rats in the EP-W stress group $(* P<0 \cdot 05)$ and the 4- to 67-week-old rats in the W-EP stress group $\left({ }^{*} P<0 \cdot 01\right.$; Fig. 1A), indicating that memory retrieval was impaired in most groups except the 4- and 7-week-old rats in EP-W group and the 76-week-old rats in the W-EP group. However, when the animals were water maze stress-experienced, memory retrieval was only impaired compared with controls by EP-W and W-EP stress in rats older than 13 weeks. Surprisingly, memory retrieval was even enhanced in 4- and 13-week-old young rats by W-EP stress compared with controls ( ${ }^{*} P<0 \cdot 05$; Fig. $\left.1 \mathrm{~B}\right)$. Although the motion performance of old animals was increased by previous water maze stress experience, for the most part this parameter did not differ between W-EP, EP-W and Control groups in water maze naive $\left(\mathrm{F}_{2,105}=0.379, P>0.05\right.$; Fig. $\left.1 \mathrm{C}\right)$ or water maze stressexperienced rats during the probe trial $\left(F_{2,105}=0 \cdot 109\right.$, $P>0 \cdot 05$; Fig. 1D) (by one-way ANOVA). The exceptions were the 67-week-old rats of the W-EP group and the 76-week-old rats of the EP-W group in water maze naive animals $\left({ }^{*} P<0 \cdot 05\right.$; Fig. $\left.1 \mathrm{C}\right)$, and the 7 -week-old rats of the EP-W and W-EP groups, and the 20- and 76-week-old rats of the W-EP group in water maze stress-experienced animals $\left({ }^{*} P<0 \cdot 05\right.$; Fig. 1D) (by repeated measures ANOVA) compared with their same age controls. Furthermore, repeated measures ANOVA showed that EP stress did not affect the ability of all groups to learn the task and there was no difference in escape latencies in the eight sessions $(P>0.05)$ except in the oldest rats (76 weeks old). Thus, EP stress affected specific aspects of spatial memory in the probe trial in almost all groups of rats.

Further comparison of these data is shown in Fig. 1E-G. Memory retrieval was much better in the 67- and 76week-old water maze stress-experienced controls compared with their water maze naive controls $\left({ }^{*} P<0 \cdot 05\right.$; Fig. 1E). Ten-week-old water maze naive animals performed the probe trial better than the 13-week-old water maze stress-experienced animals, possibly due to the different ages of the two groups $\left({ }^{*} P<0 \cdot 05\right.$; Fig. 1E). Furthermore, in the EP-W group a significantly better performance in the probe trial was found in 67- and 76-week-old water maze stress-experienced animals compared with the water maze naive animals, but the 4-weekold water maze stress-experienced animals performed the probe trial slightly worse than the water maze naive animals $\left({ }^{*} P<0 \cdot 05\right.$; Fig. $\left.1 F\right)$. However, in the W-EP group water maze stress-experienced animals performed the memory retrieval task significantly better than the water maze naive animals at all ages except 76 weeks old $\left({ }^{*} P<0 \cdot 05\right.$; Fig. $\left.1 \mathrm{G}\right)$. Thus, water maze stress experience induced enhancement of memory retrieval in old controls (Fig. 1E) and enhanced the effect of later W-EP stress on memory retrieval in 4- and 13-week-old young animals (Fig. 1B).

The above results suggest that memory retrieval is more sensitive to the W-EP stress protocol both in water maze stress-experienced and naive animals (Fig. 1A and $1 \mathrm{G}$ ). Previous water maze stress experience switches impairment of memory retrieval to enhancement in W-EP young groups. Based on the above findings, the following experiments focused on 4-week-old young and 20-weekold animals to further compare the effect of previous stress experience on memory retrieval.

\section{$E P$, foot shock or corticosterone treatment before or during training prevented the impairment effect of a later stress on memory retrieval}

In the second experiment, 4-week-old young water maze naive rats received EP stress or corticosterone $(5 \mathrm{mg} / \mathrm{kg}$, s.c.; Sigma) immediately after the final trial of six-trials training (6t-EP, 6t-Cort). Memory retrieval was significantly impaired compared with controls $\left(\mathrm{F}_{2,36}=4 \cdot 39\right.$, $* P<0 \cdot 05$; Fig. 2A). Similar results were found in the 20 -week-old rats $\left(\mathrm{F}_{2,21}=8 \cdot 472, * P<0.01\right.$; Fig. 2B). When EP stress was given immediately after each trial (6t/6EP) or corticosterone $(5 \mathrm{mg} / \mathrm{kg}$, s.c.) was given before the first trial of six-trials training (Cort-6t-Cort), memory retrieval was no longer impaired by subsequent EP stress or corticosterone $(5 \mathrm{mg} / \mathrm{kg}$, s.c.) in young animals $\left(\mathrm{F}_{2,33}=0.704, P>0 \cdot 05\right.$; Fig. 2C). No significant impairment was found with the same treatment in 20week-old rats (Fig. 2D). Again, neither of the treatments altered average swimming speed in young $\left(\mathrm{F}_{2,36}=1 \cdot 269\right.$, $P>0.05)$ and old rats $\left(F_{2,33}=0.802, P>0.05\right)$ in the probe trial. These results suggest that stress experience or elevated corticosterone resists the impairment induced by a later stress or corticosterone treatment in young animals and the resistance in adult rats was slightly decreased compared with young animals. To further understand whether the adaptation of the animals to stress contributed to the stress-experienced effect on memory retrieval, 

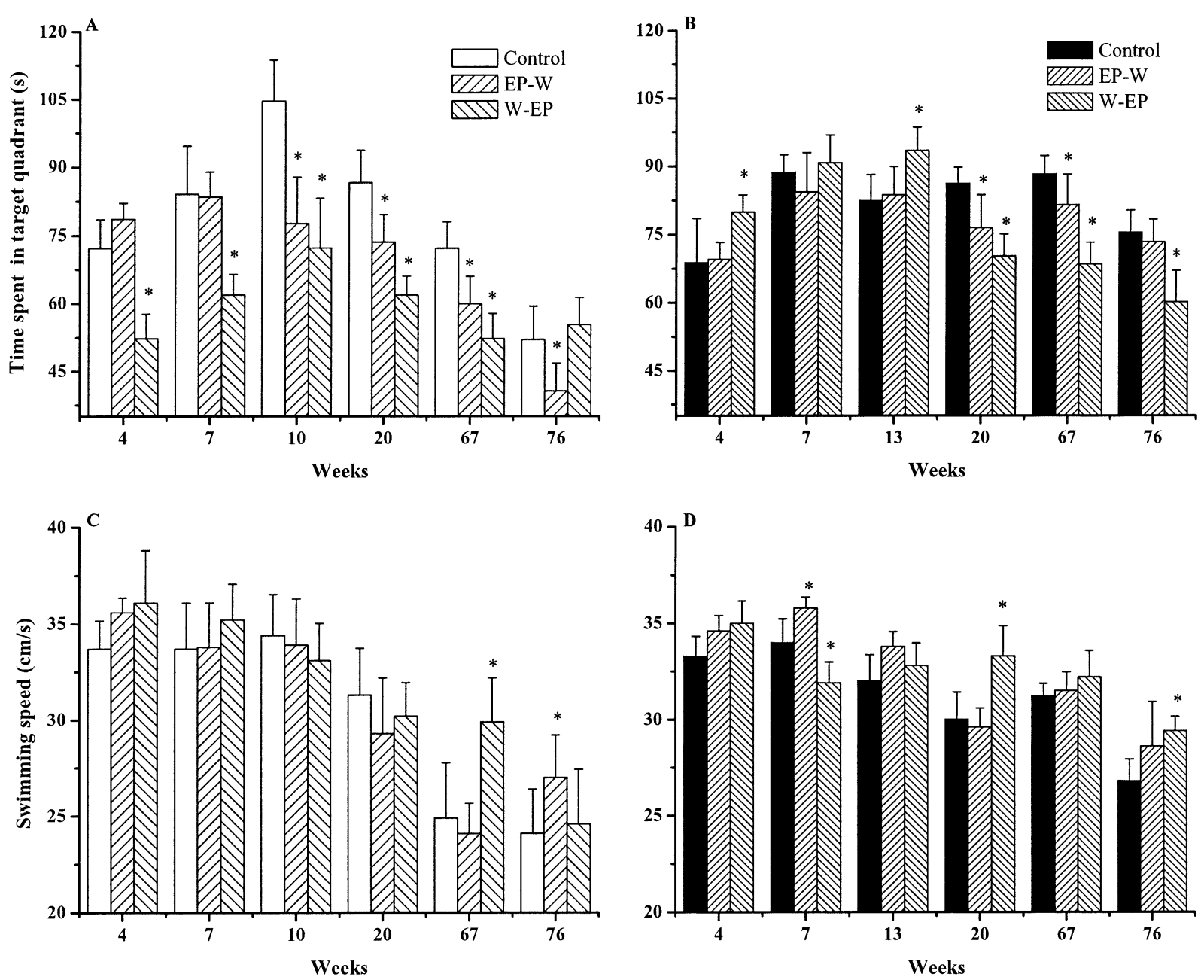

Figure 1A-D.

inescapable foot shock (a strong stress) was used in the following experiments. As expected, memory retrieval was significantly impaired both in the young and adult rats by one set of three foot shocks $(0.8 \mathrm{~mA}$ for $1 \mathrm{~s}$ with a $5-\mathrm{s}$ inter-shock interval in an operant test chamber) $30 \mathrm{~min}$ before the probe trial (6t-F) compared with controls $\left({ }^{*} P<0 \cdot 05\right.$; Fig. $2 \mathrm{E}$ and F). However, such memory impairment was prevented in young rats pre-stressed with the same foot shocks before six-trials training $(P>0 \cdot 05)$, but not in the adult animals $(* P<0 \cdot 05)$. The fact that performance in the probe trial was enhanced rather than impaired by EP stress when the animals were pre-stressed with one set of three foot shocks $\left({ }^{*} P<0 \cdot 05\right.$; Fig. $\left.2 \mathrm{G}\right)$ before six-trials training indicated that stress experience with one type of stress could also change the effects on memory of another type of stress. Such stress-experienced effects were similar when three or six sets of three foot shocks were given at $30 \mathrm{~min}$ or $24 \mathrm{~h}$ before the six-trials training (Fig. 2H).
The effect of stress experience on spatial memory is dependent on glucocorticoid receptor activation

The third experimental protocol with six-trials training was used to examine the effects of multiple stress experience on memory. Water maze stress experience prevented memory impairment $\left(\mathrm{F}_{1,24}=1 \cdot 156, P>0 \cdot 05\right.$; Fig. 3A) caused by EP stress 30 min before the probe trial, except in the 20-week-old animals $\left(\mathrm{F}_{1,14}=0 \cdot 154, * P<0 \cdot 05\right.$; Fig. $3 \mathrm{~B})$. When EP stress was given immediately after each trial, the performance in the probe trial was hardly impaired by EP stress in adult water maze stressexperienced animals $(P>0 \cdot 05$; Fig. 3D) but it was enhanced in young water maze stress-experienced animals compared with controls $\left(\mathrm{F}_{1,18}=0 \cdot 062,{ }^{*} P<0 \cdot 05\right.$; Fig. $\left.3 \mathrm{C}\right)$. In addition, RU $38486(20 \mathrm{mg} / \mathrm{kg}$, s.c.), an antagonist of glucocorticoid receptors, when given before the first trial of six-trials training, prevented the enhancement of memory (seen in Fig. 3C) caused by EP stress given immediately 

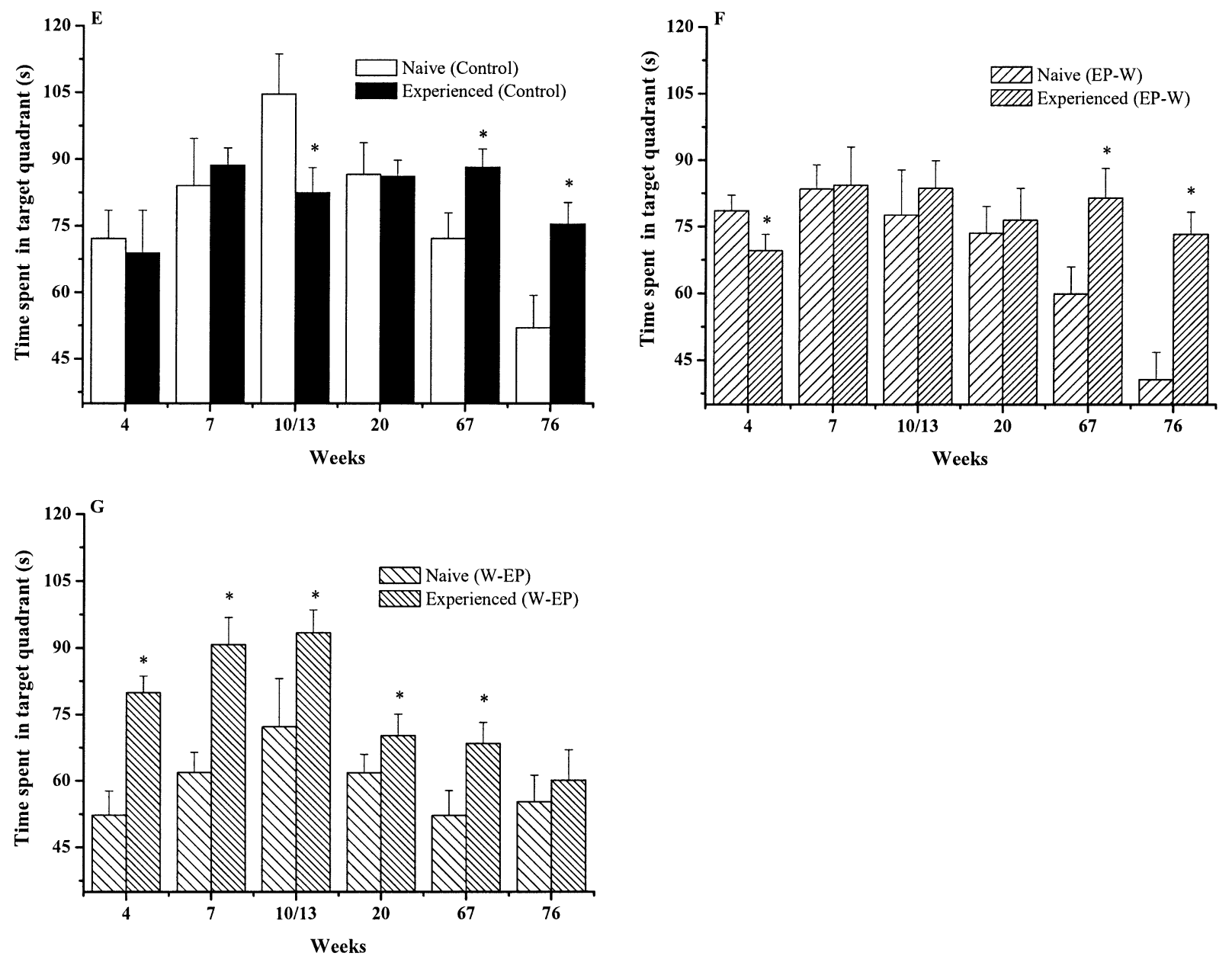

Figure $1 \mathrm{E}-\mathrm{G}$.

Figure 1 Effects of previous water maze stress experience and EP stress during training on spatial memory in different age-groups of rats. (A) Water maze naive rats. Performance in the probe trial was impaired by EP stress given either before or after each session compared with controls. (B) Water maze stress-experienced rats. The same stress protocol enhanced spatial memory in 4- and 13-week-old young rats in W-EP. (C) Total path lengths during $3 \mathrm{~min}$ of the probe trials plotted as average swimming speed $(\mathrm{cm} / \mathrm{s})$. EP stress did not affect the motion performance of the rats except the 67- and 76-week-old rats. (D) Motion performance was increased in the rats older than 13 weeks by water maze stress experience. (E) Comparison between water maze naive and water maze stress-experienced controls. (F) Comparison between water maze naive and water maze stress-experienced EP-W groups. (G) Comparison between water maze naive and water maze stress-experienced W-EP groups. Results are means \pm S.E.M., $n=6$ in each group. ${ }^{*} P<0 \cdot 05$ compared with the same age controls. Naive $=$ water maze naive; Experienced $=$ water maze stress experienced.

after each trial, in the young water maze stress-experienced rats. RU $38486(\mathrm{Ru}-6 \mathrm{t})$ and vehicle, given immediately before the first trial of six-trials training, did not affect the performance $\left(\mathrm{F}_{2,26}=0 \cdot 134, P>0 \cdot 05\right.$; Fig. $\left.3 \mathrm{E}\right)$.

\section{The effects of stress experience on plasma corticosterone levels}

Water maze stress experience induced larger decreases in plasma corticosterone levels of 4-week-old young animals compared with 10-/13- and 76-week-old animals (Table $1 \mathrm{a}$ and $\mathrm{b}$ ). However, raised plasma corticosterone levels did not prevent the lower impairment or enhancement effects of stress experience on memory retrieval in control 76-week-old animals (Fig. 1E and Table 1a), in 13-weekold W-EP-treated animals (Fig. 1B and Table 1a), in 4-week-old 6t/6EP-treated animals (Fig. 2C and Table 1c) and in 4-week-old Cort-6t-Cort-treated animals (Fig. 2C and Table 1d).

\section{Discussion}

The present finding that corticosterone treatment or stress experience before or during training induces less 


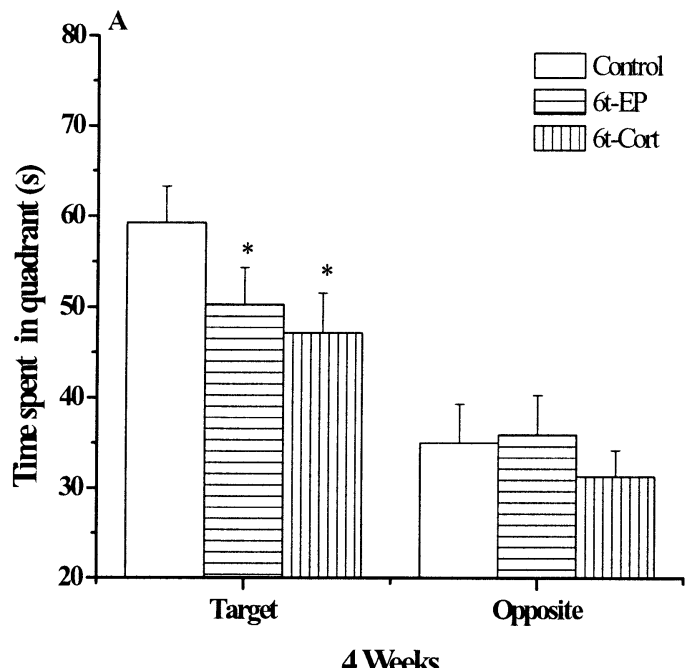

4 Weeks

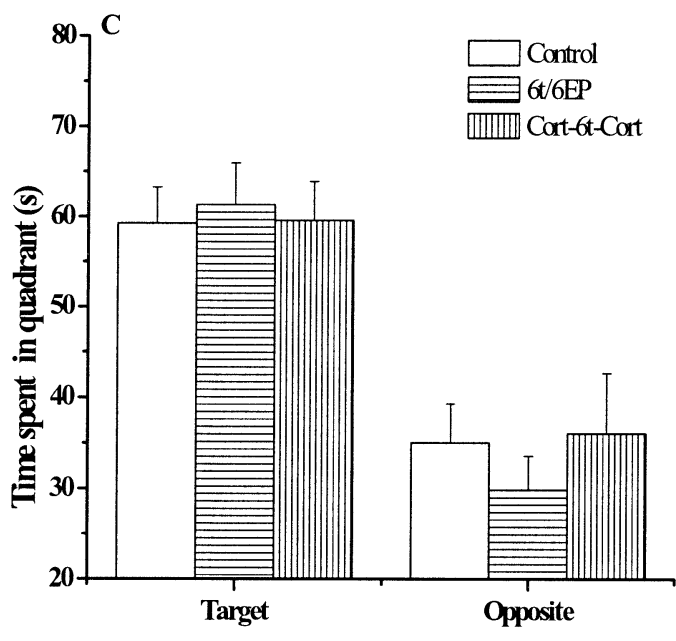

4 Weeks
B

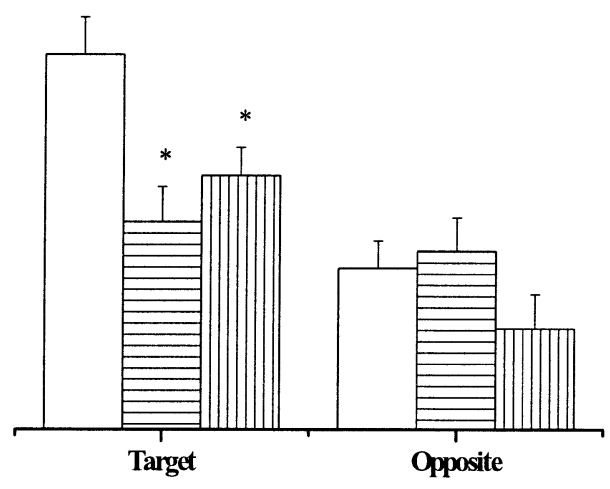

20 Weeks

D

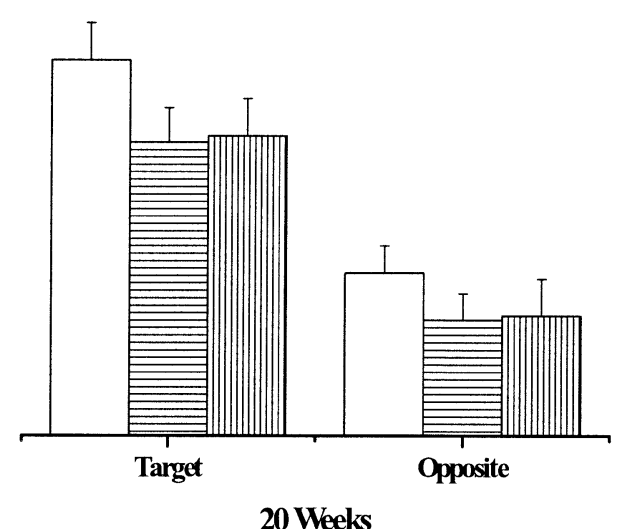

20 Weeks

Figure 2 A-D.

impairment or enhancement of memory retrieval by a later corticosterone treatment or stress before the probe trial in young but not in older animals reveals that stress experience and age are the important factors controlling the effects of behavioral stress on memory. The more stress experienced before or during behavioral training (e.g. water maze stress experience plus EP or F stress), the less impairment or enhancement of memory retrieval is caused by a later behavioral stress. Such an effect of stress experience on memory is dependent on the activation of the glucocorticoid receptor and on the age of the animals, which switches the effect of stress on memory to impairment or enhancement.
Impairment or enhancement effect of stress on memory is determined by stress experience and age

Experiments with the first protocol showed that the performance in the probe trial decreased following ontogeny in water maze naive controls; meanwhile memory retrieval was profoundly impaired by EP stress given either before or after each session $\left({ }^{*} P<0 \cdot 05\right.$; Fig. $\left.1 \mathrm{~A}\right)$. When the animals had previously experienced water maze stress for five days, the performance in the probe trial was no longer different between young and old animals, and EP stress still impaired the performance of the animals older than 13 weeks $\left({ }^{*} P<0 \cdot 05\right.$; Fig. 1B). However, memory retrieval 


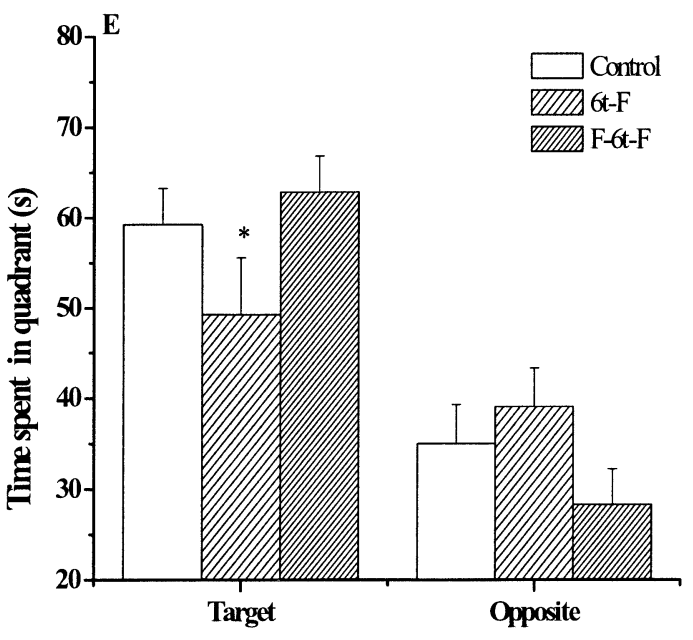

4Weeks

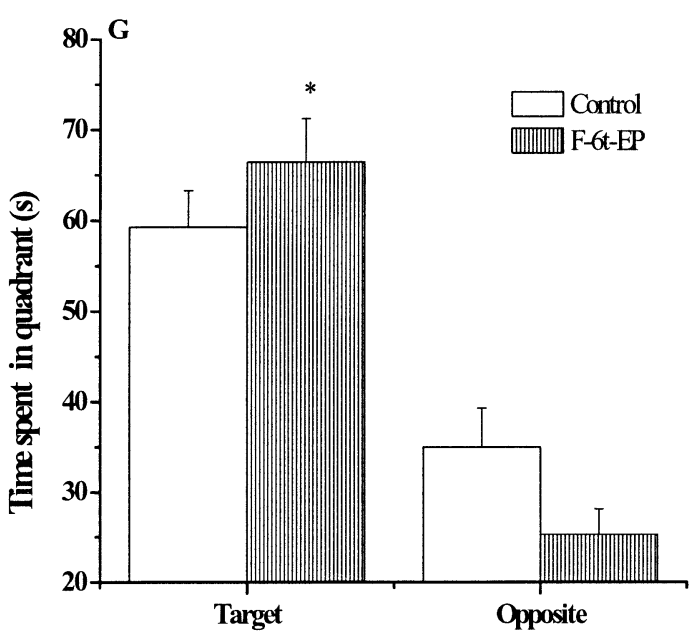

4 Weeks
$\mathbf{F}$

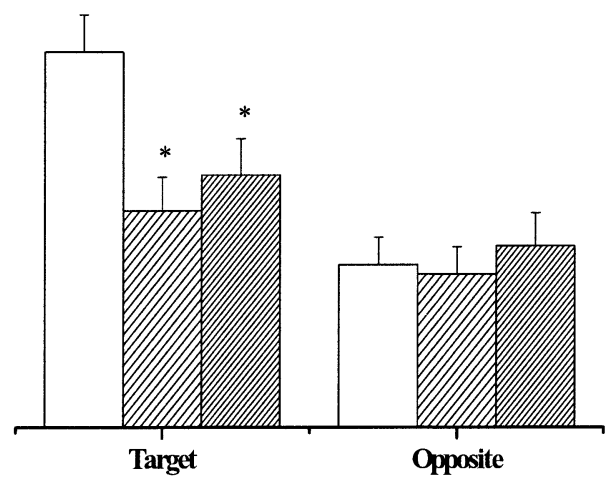

20 Weeks

$\mathbf{H}$

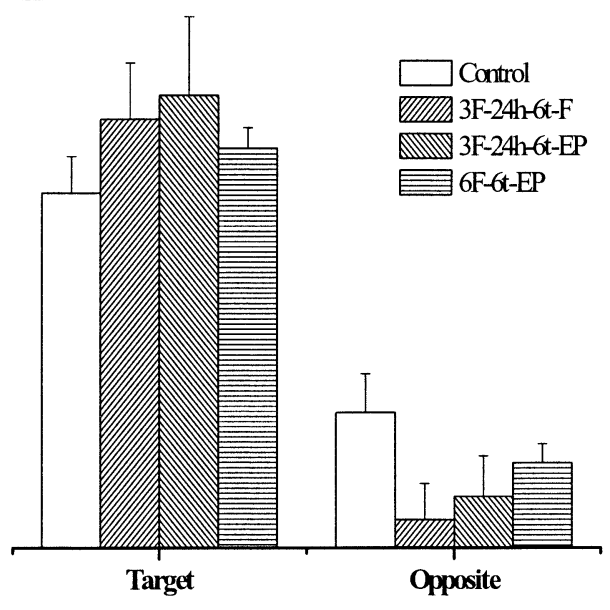

4 Weeks

Figure $2 \mathrm{E}-\mathrm{H}$.

Figure 2 Elevated platform (EP), foot shock (F) or corticosterone (Cort) treatment before or during training prevented the impairment effect of a later stress on memory retrieval in water maze naive rats. Target $=$ target quadrant; Opposite=opposite quadrant. (A) EP stress (6t-EP, $n=12)$ or corticosterone $(5 \mathrm{mg} / \mathrm{kg}$, s.c; $6 \mathrm{t}$-Cort, $n=9)$ significantly impaired memory retrieval in 4-week-old rats compared with controls $(n=18)$. (B) Similar results were found in 20-week-old rats with the same protocol (Control, $n=11 ; 6 \mathrm{t}$-EP, $n=7 ; 6 \mathrm{t}$-Cort, $n=6$ ). (C) When EP stress was given immediately after each trial or corticosterone $(5 \mathrm{mg} / \mathrm{kg}$, s.c) treatment was given before the six-trials training, memory retention in the probe trial was no longer impaired by EP stress (6t/6EP, $n=10)$ or corticosterone $(5 \mathrm{mg} / \mathrm{kg}$, s.c; Cort- $6 \mathrm{t}$-Cort, $n=8)$ compared with controls $(n=18)$ in young animals. (D) No significant impairment was found in the 20-week-old animals (Control, $n=11 ; 6 \mathrm{t} / 6 \mathrm{EP}, n=7$; Cort-6t-Cort, $n=6$ ). (E) In young animals that received one set of three foot shocks $30 \mathrm{~min}$ before the probe trial, memory retrieval was significantly impaired $(6 t-F, n=8)$. Pre-stressing the animals with one set of three foot shocks before training prevented the impairment of memory retention induced by one set of three foot shocks after training (F-6t-F, $n=7$; Control, $n=18$ ). (F) Similar but greater impairment was found in the 20-week-old animals $(6 t-F, n=7)$. Pre-stressing animals with foot shocks did not prevent the impairment induced by later foot shocks (F-6t-F, $n=7$; Control, $n=11$ ). (G) EP stress 30 min before the probe trial enhanced memory retention when the young animals received foot shocks before the training (F-6t-EP, $n=7$; Control, $n=18$ ). (H) The effects were similar when the young animals received one set of three foot shocks $24 \mathrm{~h}$ (3F-24 h-6t-F, $n=6$; $3 \mathrm{~F}-24 \mathrm{~h}-6 \mathrm{t}-\mathrm{EP}$, $n=6$ ) or two sets of three foot shocks $30 \mathrm{~min}$ (6F-6t-EP, $n=6$; Control, $n=18$ ) before the training. ${ }^{*} P<0 \cdot 05$ compared with controls. 


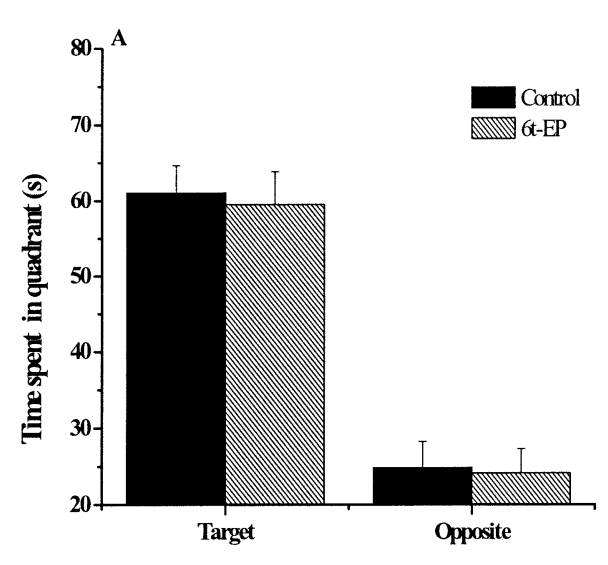

4 Weeks

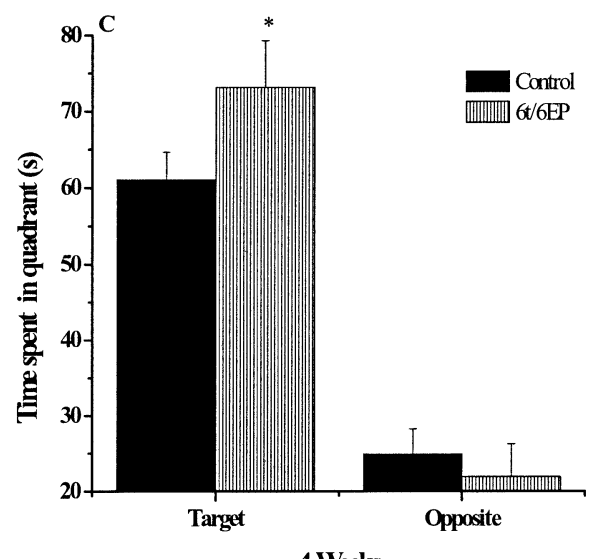

4 Weeks
B

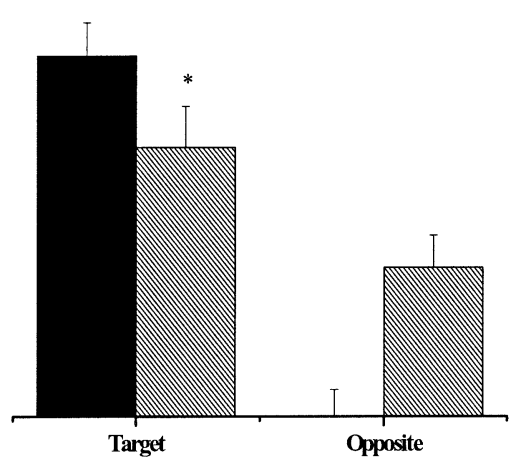

20 Weeks

D

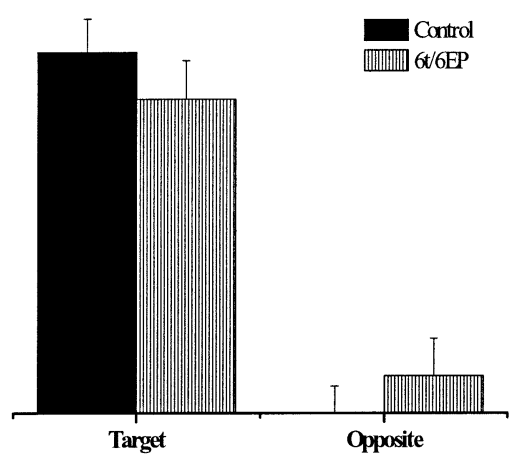

20 Weeks

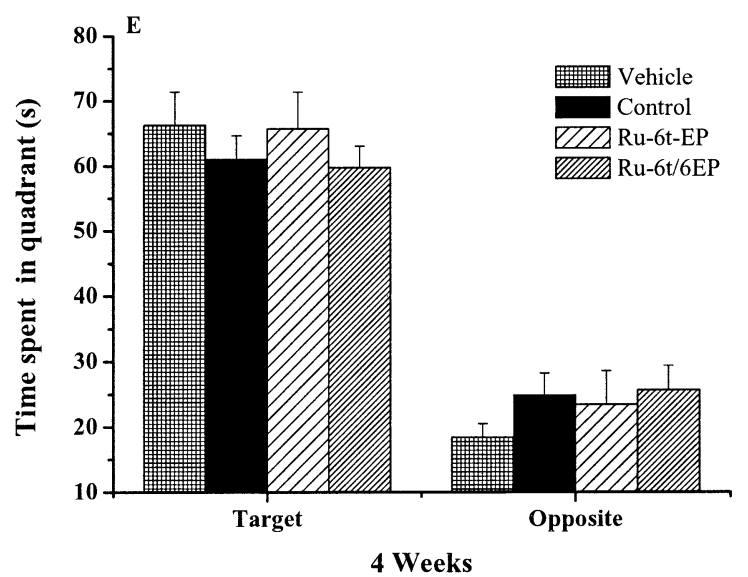

Figure 3 The EP stress enhanced performance of young water maze stress-experienced animals in the probe trial was dependent on glucocorticoid receptors. (A) EP stress 30 min before the probe trial did not affect memory retention in the 4-week-old young water maze stress-experienced animals (6t-EP, $n=6$; Control, $n=12$ ). (B) EP stress still impaired memory retention in 20-week-old water maze stress-experienced animals (6t-EP, $n=8$; Control, $n=8)$. (C) When EP stress was given after each trial, performance in the probe trial was significantly enhanced (6t/6EP, $n=8$; Control, $n=12$ ). (D) No significant impairment was found in 20-week-old animals under the same condition compared with controls (Control, $n=8 ; 6 \mathrm{t} / 6 \mathrm{EP}, n=8)$. (E) The glucocorticoid receptor antagonist RU $38486(20 \mathrm{mg} / \mathrm{kg}$, s.c.) prevented the enhancement of memory retention caused by EP stress given after each trial (RU-6t/6EP, $n=6)$. RU 38486 (20 mg/kg, s.c.) and vehicle itself did not affect the performance in the probe trial (Ru-6t-EP, $n=11$; Control, $n=12 ;$ Vehicle, $n=8$ ). ${ }^{\star} P<0 \cdot 05$. 
Table 1 Effects of stress-experience on plasma corticosterone levels $(\mu \mathrm{g} / \mathrm{dl})$. Results are means \pm S.E.M.

Age (weeks)

Treatment

(a) Water maze stress-experienced

Control

EP-W

W-EP

(b) Water maze naive

Control

EP-W

W-EP

(c) Water maze stress-experienced

Control

6t-EP

$6 \mathrm{t} / 6 \mathrm{EP}$

RU-6t/6EP

(d) Water maze naive

Vehicle

6t-Cort

Cort-6t-Cort

\begin{tabular}{|c|c|c|}
\hline 4 & 13 & 76 \\
\hline $1 \cdot 5 \pm 0.7$ & $11 \cdot 4 \pm 0 \cdot 8$ & $22 \cdot 1 \pm 1 \cdot 5$ \\
\hline $0.6 \pm 0.3$ & $14 \cdot 7 \pm 2 \cdot 0$ & $21 \cdot 3 \pm 4 \cdot 1$ \\
\hline $2 \cdot 8 \pm 0 \cdot 7$ & $21 \cdot 5 \pm 2 \cdot 6$ & $24 \cdot 7 \pm 3 \cdot 8$ \\
\hline 4 & 10 & 76 \\
\hline $1.0 \pm 0 \cdot 2$ & $23 \cdot 8 \pm 3 \cdot 4$ & $30 \cdot 6 \pm 8 \cdot 3$ \\
\hline $11 \cdot 5 \pm 2 \cdot 6$ & $25 \cdot 0 \pm 2 \cdot 4$ & $28 \cdot 5 \pm 9 \cdot 1$ \\
\hline $22 \cdot 2 \pm 2 \cdot 0$ & $19 \cdot 5 \pm 2 \cdot 2$ & $36 \cdot 6 \pm 5 \cdot 7$ \\
\hline
\end{tabular}

\begin{tabular}{|c|c|}
\hline 4 & 20 \\
\hline $1 \cdot 9 \pm 0 \cdot 3$ & $3 \cdot 7 \pm 0 \cdot 2$ \\
\hline $17 \cdot 4 \pm 1 \cdot 7$ & $12 \cdot 8 \pm 0 \cdot 9$ \\
\hline $8 \cdot 1 \pm 0 \cdot 7$ & $18 \cdot 0 \pm 1 \cdot 5$ \\
\hline $9 \cdot 6 \pm 0 \cdot 9$ & \\
\hline
\end{tabular}

$3 \cdot 8 \pm 1 \cdot 5$

$35 \cdot 4 \pm 3 \cdot 4$

$46 \cdot 1 \pm 9 \cdot 3$

Plasma corticosterone levels of groups shown in Fig. 1B(a), Fig. 1A(b), Fig. 3(c) and Fig. 2A and C(d). Sample size is 6 individuals per group except Vehicle $(n=4)$, 6t-Cort $(n=4)$ and Cort-6t-Cort $(n=10)$.

was surprisingly enhanced in 4- and 13-week-old animals by W-EP stress, indicating two possibilities: either there was an adaptation process due to the previous five days of water maze stress experience or there was a build up of resistance to a later stress. Five days handling without any behavior training is also a stressful experience as shown by the different effect on plasma corticosterone levels found in 4- and 13-week-old animals $(n=4,1 \cdot 9 \pm 0 \cdot 3 \mu \mathrm{g} / \mathrm{dl}$; $9 \cdot 9 \pm 0.6 \mu \mathrm{g} / \mathrm{dl}$ ), indicating that handling itself is another mild stress experienced by all groups of animals. In the animals in the behavioral training experiments, plasma levels of corticosterone were decreased in the water maze stress-experienced groups (EP-W, W-EP and Controls) and a larger reduction was found in the 4-week-old young animals compared with water maze naive animals (Table $1 \mathrm{a}$ and $1 \mathrm{~b}$ ). These results indicate that the water maze is very stressful to animals and that adaptation occurred in the experiment, in agreement with previous studies (Cizza et al. 1995, Conrad et al. 1996, Mabry et al. 1996). The decreased levels of plasma corticosterone induced by water maze stress experience may also be due to the build up of resistance to a later stress. However, it could not explain why elevated platform stress can enhance the retrieval of spatial memory in young animals that were water maze stress experienced. Furthermore, pre-treatment with corticosterone or EP stress after each trial in the second protocol prevented the impairment of spatial memory induced by corticosterone or EP stress $30 \mathrm{~min}$ before the probe trial (Fig. 2C and 2D) even though plasma corti- costerone levels were raised (Table 1c and 1d), consistent with previous findings (Sandi et al. 1997). Thus, preactivation of glucocorticoid receptors rather than the plasma corticosterone concentrations may, at least in part, determine the effects of stress on memory which not only prevents the decrease in memory retrieval during ontogeny but also enhances memory in young animals (Fig. 1B, 3C and 3D). Since many forms of experiencedependent memory have been found, a possible alternative explanation could be that previous stress experience triggers the processes of an aberrant form of stress memory that interacts with the behavioral training and results in less impairment or enhancement effects of stress in the behavioral tests.

Stress experience changes the sensitivity of spatial memory to a later stress

The sensitivity of memory to stress follows an inverted U-shaped dose-response relationship (McEwen \& Sapolsky 1995, Kim \& Yoon 1998, Conrad et al. 1999, de Kloet et al. 1999, Sapolsky 2000, Lupien \& Lepage 2001) and activation of glucocorticoid receptors seems to be a prerequisite for the long-term storage of information. Our findings indicate that stress experience is a key factor governing the sensitivity of age-related memory to a later stress, in which the curve of the dose-response may be shifted in young animals but is little affected in older animals, indicating that an age older than 13 weeks is the 
truly sensitive age when memory becomes more vulnerable to repetitive daily stress. Such effects of stress experience may relate to the effects of stress on metaplasticity (Kim \& Yoon 1998, McEwen 1999) in which hippocampal synaptic plasticity was age dependent (Bear \& Abraham 1996, Wagner \& Alger 1996). Our results are important in considering the fact that corticosteroid hormones are necessary and have a positive effect at some time points but are regarded as disruptive for memory at other time points (Lupien \& Lepage 2001). Our results are even more important in considering the fact that stress memory induced by stress experience (e.g. an aberrant form of stress memory in post-traumatic stress disorder, depression etc.) interacts with the water maze training memory and results in less impairment or enhancement effects on memory caused by a later stress, which may be due to the effects of glucocorticoid receptor activation and the changes during ontogeny (such as feedback inhibition, cognition reduction in ageing, capacity to withstand stress etc.). It is highly possible that individual cognitive function under stressful events will be dependent on their previous stress experience and age.

\section{Acknowledgements}

National Basic Research Program G1999054000, National Natural Science grants 39870280, 39925011, 39930080 and Yunnan Science Foundation grant 98C111 M of China to L X supported this work.

\section{References}

Balfour DJ \& Reid A 1979 Effects of betamethasone on the stimulation of corticosterone secretion in rats. Archives Internationales de Pharmacodynamie et de Therapie 237 67-74.

Bear MF \& Abraham WC 1996 Long-term depression in hippocampus. Annual Reviews in Neuroscience 19 437-462.

Bodnoff SR, Humphreys AG, Lehman JC, Diamond DM, Rose GM \& Meaney MJ 1995 Enduring effects of chronic corticosterone treatment on spatial learning, synaptic plasticity, and hippocampal neuropathology in young and mid-aged rats. Journal of Neuroscience 15 61-69.

Cizza G, Pacak K, Kvetnansky R, Palkovits M, Goldstein DS, Brady LS, Fukuhara K, Bergamini E, Kopin IJ, Blackman MR et al. 1995 Decreased stress responsivity of central and peripheral catecholaminergic systems in aged 344/N Fischer rats. Journal of Clinical Investigation 95 1217-1224.

Conrad CD, Galea LA, Kuroda Y \& McEwen BS 1996 Chronic stress impairs rat spatial memory on the $\mathrm{Y}$ maze, and this effect is blocked by tianeptine pretreatment. Behavioral Neuroscience 110 1321-1334.

Conrad CD, Lupien SJ \& McEwen BS 1999 Support for a bimodal role for type II adrenal steroid receptors in spatial memory. Neurobiology of Learning and Memory 72 39-46.

Diamond DM, Park CR, Heman KL \& Rose GM 1999 Exposing rats to a predator impairs spatial working memory in the radial arm water maze. Hippocampus 9 542-552.

Ferry B, Roozendaal B \& McGaugh JL 1999 Role of norepinephrine in mediating stress hormone regulation of long-term memory storage: a critical involvement of the amygdala. Biological Psychiatry 46 1140-1152.
Flood JF, Bennett EL, Rosenzweig MR \& Orme AE 1972 Influence of training strength on amnesia induced by pretraining injections of cycloheximide. Physiology and Behavior 9 589-600.

Garcia R 2001 Stress, hippocampal plasticity, and spatial learning. Synapse 40 180-183.

Garcia R, Paquereau J, Vouimba RM \& Jaffard R 1998 Footshock stress but not contextual fear conditioning induces long-term enhancement of auditory-evoked potentials in the basolateral amygdala of the freely behaving rat. European Journal of Neuroscience 10 457-463.

Kim JJ \& Yoon KS 1998 Stress: metaplastic effects in the hippocampus. Trends in Neurosciences 21 505-509.

de Kloet ER, Oitzl MS \& Joels M 1999 Stress and cognition: are corticosteroids good or bad guys? Trends in Neurosciences $\mathbf{2 2}$ $422-426$.

Luine V, Martinez C, Villegas M, Magarinos AM \& McEwen BS 1996 Restraint stress reversibly enhances spatial memory performance. Physiology and Behavior 59 27-32.

Lupien SJ \& Lepage M 2001 Stress, memory, and the hippocampus: can't live with it, can't live without it. Behavioural Brain Research 127 137-158.

Mabry TR, McCarty R, Gold PE \& Foster TC 1996 Age and stress history effects on spatial performance in a swim task in Fischer-344 rats. Neurobiology of Learning and Memory 66 1-10.

McEwen BS 1999 Stress and hippocampal plasticity. Annual Reviews in Neuroscience 22 105-122.

McEwen BS 2000 Effects of adverse experiences for brain structure and function. Biological Psychiatry 48 721-731.

McEwen BS \& Sapolsky RM 1995 Stress and cognitive function. Current Opinion in Neurobiology 5 205-216.

McEwen BS, Albeck D, Cameron H, Chao HM, Gould E, Hastings N, Kuroda Y, Luine V, Magarinos AM, McKittrick CR et al. 1995 Stress and the brain: a paradoxical role for adrenal steroids. Vitamins and Hormones 51 371-402.

McGaugh JL, Cahill L \& Roozendaal B 1996 Involvement of the amygdala in memory storage: interaction with other brain systems. PNAS 93 13508-13514.

Morris R 1984 Developments of a water-maze procedure for studying spatial learning in the rat. Journal of Neuroscience Methods 11 47-60.

Oitzl MS, Fluttert M, Sutanto W \& de Kloet ER 1998 Continuous blockade of brain glucocorticoid receptors facilitates spatial learning and memory in rats. European Journal of Neuroscience 10 3759-3766.

Payne JD, Nadel L, Allen JJ, Thomas KG \& Jacobs WJ 2002 The effects of experimentally induced stress on false recognition. Memory $101-6$.

de Quervain DJ, Roozendaal B \& McGaugh JL 1998 Stress and glucocorticoids impair retrieval of long-term spatial memory. Nature $394787-790$

Sandi C, Loscertales M \& Guaza C 1997 Experience-dependent facilitating effect of corticosterone on spatial memory formation in the water maze. European Journal of Neuroscience 9 637-642.

Sapolsky RM 2000 Stress hormones: good and bad. Neurobiology of Disease 7 540-542.

Shors TJ \& Servatius RJ 1997 The contribution of stressor intensity, duration, and context to the stress-induced facilitation of associative learning. Neurobiology of Learning and Memory 68 92-96.

Shors TJ, Weiss C \& Thompson RF 1992 Stress-induced facilitation of classical conditioning. Science 257 537-539.

Wagner JJ \& Alger BE 1996 Homosynaptic LTD and depotentiation: do they differ in name only? Hippocampus 6 24-29.

Xu L, Anwyl R \& Rowan MJ 1997 Behavioural stress facilitates the induction of long-term depression in the hippocampus. Nature 387 497-500.

Received in final form 1 April 2003

Accepted 4 April 2003 